\title{
Stacking a 4D geometry into an Einstein-Gauss-Bonnet bulk
}

\author{
Carlos Barceló, Roy Maartens, Carlos F. Sopuerta, and Fermín Viniegra \\ Institute of Cosmology and Gravitation, University of Portsmouth, Portsmouth PO1 2EG, Britain
}

\begin{abstract}
In Einstein gravity there is a simple procedure to build $D$-dimensional spacetimes starting from $(D-1)$-dimensional ones, by stacking any $(D-1)$-dimensional Ricci-flat metric into the extradimension. We analyze this procedure in the context of Einstein-Gauss-Bonnet gravity, and find that it can only be applied to metrics with a constant Krestschmann scalar. For instance, we show that solutions of the black-string type are not allowed in this framework.
\end{abstract}

PACS numbers: 04.50.+h, 11.25.Mj, 11.25.Db

\section{INTRODUCTION}

One of the main ingredients in most attempts to unify the four fundamental interactions known at present in nature is the existence of additional dimensions. If there is a limit in which one can make sense of these additional dimensions classically, it will be fundamentally important to know what the field equations are that govern the higher-dimensional spacetime. Lovelock [1] proved that for an arbitrary number of dimensions, the most general classical gravitational Lagrangian with associated field equations $\mathcal{G}_{A B}=0$, such that $\mathcal{G}_{A B}$ is symmetric, divergence-free, and constructed with up to second derivatives of the metric, is formed by a linear combination (with arbitrary coefficients) of the dimensionally-extended Euler densities. As is well known, in four dimensions there are only two Euler densities that are not topological invariants, and therefore have a non-trivial dynamical content. They are the scalar curvature term and the cosmological constant term in the Einstein-Hilbert action. Experimentally it has been possible to determine the values of their two associated coefficients: Newton's constant and cosmological constant. In $D=5$ and beyond there are additional Euler densities that have to be considered. The relative weight of these additional terms in the dynamics of the system is something to be determined experimentally.

From our 4-dimensional point of view, the higher-dimensional geometries of more immediate interest are those suitable for a standard or exotic Kaluza-Klein reduction. In this paper we will consider only 5-dimensional geometries of this type. In five dimensions there is only one additional Euler density to be considered: the Gauss-Bonnet term. Our main motivation for studying 5-dimensional geometries with a Gauss-Bonnet term comes about from the exotic Kaluza-Klein reduction realized in the Randall-Sundrum braneworld scenario [2, 3]. In this scenario our universe is described as a 4-dimensional brane immersed in a 5-dimensional anti-de Sitter bulk. When the braneworld scenario is considered as a low-energy limit of string/M theory it becomes even more natural to consider the effects of the Gauss-Bonnet term [4, 5].

In the presence of Gauss-Bonnet modifications, it was first proved that it is possible to obtain a vacuum geometry equivalent to that of Randall-Sundrum apart from a redefinition of the constants [6]. This redefinition is such that it allows a Minkowski brane in an anti-de Sitter bulk even without a bulk negative cosmological constant and a positive brane tension [7]. A massless graviton is also shown to appear in this construction 8, 9]. In the context of self tuning mechanisms for the vanishing of the cosmological constant (see for example 10] and references therein), the Gauss-Bonnet term allows to avoid the presence of naked singularities in the bulk 11]. Cosmology on the brane in this scenario has received much attention [12, 13, 14, 15, 16, 17]. In all these works the brane is maximally symmetric or of cosmological type. Here, we analyze branes with arbitrary metric (but with specific extensions into the bulk).

In Einstein General Relativity there is an easy procedure to produce 5-dimensional solutions of the field equations by trivially extending vacuum 4-dimensional solutions into the extra dimension [18]. This procedure was used to find a black string solution [19] and a plane wave solution [20] in the realm of braneworlds. We show that this procedure for generating solutions does not generalize to Einstein-Gauss-Bonnet gravity. For the procedure to be applicable, the 4-dimensional geometry has to satisfy an additional constraint: its Krestschmann scalar has to be constant. This implies in particular that geometries of black-string type cannot be constructed in this framework.

In the next section we review and generalize the procedure for generating $D$-dimensional solutions starting from $(D-1)$-dimensional ones. We show that the procedure works not only for vacuum solutions [18], but for Einstein manifolds. Then, in section $\amalg$ we investigate what happens in the case of Einstein-Gauss-Bonnet gravity with $D=5$. Finally, we discuss the results and conclude. 


\section{STACKING TECHNIQUE}

There is a set of solutions to $D$-dimensional Einstein gravity that can be constructed in a simple way starting from vacuum $(D-1)$-dimensional solutions. The procedure is based on the stacking of any vacuum $(D-1)$-dimensional solution into the additional dimension [18]. Given any metric $g_{\mu \nu}(x)$ such that the $(D-1)$-dimensional Einstein tensor satisfies $G_{\mu \nu}=0$, then

$$
d s^{2}=d y^{2}+g_{\mu \nu}(x) d x^{\mu} d x^{\nu}
$$

is a solution of the $D$-dimensional Einstein equations ${ }^{(D)} G_{A B}=0$. This procedure can also be used when a $D$ dimensional negative cosmological constant $\left|\Lambda_{D}\right|=(D-2)(D-1) / 2 l^{2}$ is present in the bulk: any metric ${ }^{(D)} g_{A B}$ of the form

$$
d s^{2}=d y^{2}+e^{-2 y / l} g_{\mu \nu}(x) d x^{\mu} d x^{\nu},
$$

where $g_{\mu \nu}(x)$ is a $(D-1)$-dimensional vacuum solution, is a solution of

$$
{ }^{(D)} G_{A B}=-\Lambda_{D}{ }^{(D)} g_{A B}
$$

Starting with solutions of the type (2) one can easily construct braneworld geometries by using the standard cut-and-paste procedure. For instance, the metric

$$
d s^{2}=d y^{2}+e^{-2|y| / l} g_{\mu \nu}(x) d x^{\mu} d x^{\nu}
$$

represents a braneworld geometry with $\mathbb{Z}_{2}$ symmetry with respect to the location of the brane $(y=0)$. In $D=5$, if we take $g_{\mu \nu}$ to be the 4-dimensional Schwarzschild metric we reproduce the black string geometry [19]. Instead, if we take $g_{\mu \nu}$ to be a pp-wave we have a 5-dimensional pp-wave travelling parallel to the brane [20].

This technique can be further generalized to the stacking of any Einstein manifold. Using the ansatz for the $D$-dimensional metric ${ }^{1}$

$$
d s^{2}=d y^{2}+e^{-2 A(y)} g_{\mu \nu}(x) d x^{\mu} d x^{\nu},
$$

the Einstein equations (3) can be split into

$$
G_{\mu \nu}(x)=-C_{2}(y) g_{\mu \nu}(x),
$$

which correspond to the $\mu \nu$ components, and

$$
C_{3}(y)+C_{4}(y) R(x)=0,
$$

corresponding to the $y y$ component, where $R=g^{\mu \nu} R_{\mu \nu}$. The coefficients are given by

$$
\begin{aligned}
& C_{2}(y)=\left[\Lambda_{D}-(D-2)\left(A^{\prime \prime}-\frac{(D-1)}{2} A^{\prime 2}\right)\right] e^{-2 A}, \\
& C_{3}(y)=\Lambda_{D}+\frac{(D-1)(D-2)}{2} A^{\prime 2}, \\
& C_{4}(y)=-\frac{1}{2} e^{2 A} .
\end{aligned}
$$

By differentiating equation (77) with respect to any brane coordinate $x^{\mu}$ we obtain

$$
C_{4}(y) R(x)_{, \mu}=0,
$$

so that the Ricci scalar has to be constant. For convenience we write this constant as

$$
R= \pm \frac{(D-1)(D-2)}{L^{2}}
$$

\footnotetext{
${ }^{1}$ It is interesting to note that by using additional freedom in the way in which a $(D-1)$-dimensional geometry is embedded into a $D$-dimensional Einstein manifold one can locally and isometrically embed whatever metric [21].
} 
From Eq. (17) and for a negative cosmological constant in the bulk, we have an equation for $A(y)$ of the form

$$
\frac{(D-1)(D-2)}{2}\left(-\frac{1}{l^{2}}+A^{\prime 2} \mp \frac{1}{L^{2}} e^{2 A}\right)=0 .
$$

Then Eq. (6) becomes

$$
G_{\mu \nu}(x)=\mp \frac{(D-2)(D-3)}{2 L^{2}} g_{\mu \nu}(x),
$$

which shows that $g_{\mu \nu}(x)$ must be a $(D-1)$-dimensional Einstein metric. Equation (13) can be easily solved. When $L \rightarrow \infty$ we have $A(y)=y / l+b$, i.e., the solution in Eq. (2) (the constant $b$ is irrelevant for the geometry). For $L \neq 0$ and defining $A=-\ln B$ we have

$$
B^{\prime 2}=\frac{B^{2}}{l^{2}} \pm \frac{1}{L^{2}} .
$$

The solution corresponding to the plus sign (positively curved brane) is

$$
d s^{2}=d y^{2}+\left(\frac{l}{L}\right)^{2} \sinh ^{2}\left(\frac{y-y_{0}}{l}\right) g_{\mu \nu}(x) d x^{\mu} d x^{\nu},
$$

and that corresponding to the minus sign (negatively curved brane) is

$$
d s^{2}=d y^{2}+\left(\frac{l}{L}\right)^{2} \cosh ^{2}\left(\frac{y-y_{0}}{l}\right) g_{\mu \nu}(x) d x^{\mu} d x^{\nu} .
$$

Again one can start from these bulk solutions to build braneworld models. The braneworld models with maximally symmetric branes, 4-dimensional de Sitter and anti-de Sitter branes 22], are the simplest illustration of this procedure. What we have shown here is that by using the same warp factors one can have not only maximally symmetric branes but any brane of the Einstein type.

For the case of a positive cosmological constant in the bulk, $B(y)$ satisfies

$$
B^{\prime 2}=-\frac{B^{2}}{l^{2}} \pm \frac{1}{L^{2}}
$$

which has solutions only for the plus sign (positively curved sections):

$$
B(y)=\frac{l}{L} \sin \left(\frac{y-y_{0}}{l}\right), \quad \text { or } \quad B(y)=\frac{l}{L} \cos \left(\frac{y-y_{0}}{l}\right) .
$$

The case with zero bulk cosmological constant can be obtained by taking the limit $l \rightarrow \infty$,

$$
B(y)=\frac{y-y_{0}}{L} .
$$

For completeness, it is interesting to observe that this technique can also be used to generate bulk solutions with positive cosmological constant and arbitrarily curved sections. The result in this case differs from the previous one in the following respect: given any Euclidean metric $g_{\mu \nu}(x)$ such that $R_{\mu \nu}= \pm\left[(D-2) / L^{2}\right] g_{\mu \nu}(x)$, then

$$
d s^{2}=-d t^{2}+B(t)^{2} g_{\mu \nu}(x) d x^{\mu} d x^{\nu},
$$

with

$$
\begin{aligned}
& B(t)=e^{2 t / l} \text { for } L \rightarrow \infty, \\
& B(t)=\frac{l}{L} \cosh \left(\frac{y-y_{0}}{l}\right) \quad \text { for the positive sign, } \\
& B(t)=\frac{l}{L} \sinh \left(\frac{y-y_{0}}{l}\right) \text { for the negative sign, }
\end{aligned}
$$

are Lorentzian $D$-dimensional Einstein manifolds of positive curvature. This might be of interest in standard cosmology. One can deduce this result easily from the above solutions for negative bulk cosmological constant via the substitutions: $l \rightarrow-i l, t \rightarrow i t$ and $L \rightarrow-i L$. 


\section{EINSTEIN-GAUSS-BONNET GRAVITY}

Our central aim is to investigate the stacking technique in the simplest modification of Einstein gravity for higherdimensional spacetimes, the Einstein-Gauss-Bonnet theory. From now on we will set $D=5$. The theory is defined by the action

$$
S=\frac{1}{2 \kappa_{5}^{2}} \int d x^{5} \sqrt{-{ }^{(5)} g}\left[{ }^{(5)} R-2 \Lambda_{5}+\alpha^{(5)} L_{G B}\right]
$$

where

$$
{ }^{(5)} L_{G B}={ }^{(5)} R^{A B C D(5)} R_{A B C D}-4{ }^{(5)} R^{A B(5)} R_{A B}+{ }^{(5)} R^{2},
$$

and $\alpha$ is the coupling constant. The field equations associated with the Einstein-Gauss-Bonnet action are

$$
{ }^{(5)} G_{A B}=-\Lambda_{5}{ }^{(5)} g_{A B}-\alpha^{(5)} H_{A B},
$$

with

$$
{ }^{(5)} H_{A B}=2^{(5)} R_{A C D E}{ }^{(5)} R_{B}{ }^{C D E}-4^{(5)} R_{A C B D}{ }^{(5)} R^{C D}-4{ }^{(5)} R_{A C}{ }^{(5)} R_{B}{ }^{C}+2^{(5)} R^{(5)} R_{A B}-\frac{1}{2}{ }^{(5)} g_{A B}{ }^{(5)} L_{G B} .
$$

This tensor is divergence free, $\nabla^{A}{ }^{(5)} H_{A B}=0$, and so it can be considered as some sort of source in the Einstein equations. The existence of this term has dramatic consequences for the stacking procedure.

\section{A. Stacking with a Gauss-Bonnet term}

We consider a metric of the form given in Eq. (5), and introduce it into the Einstein-Gauss-Bonnet equations. By doing this, we obtain a set of equations playing the role of the effective 4-dimensional Einstein equations [the $\mu \nu$-components of Eq. [27]],

$$
C_{1}(y) G_{\mu \nu}(x)=-C_{2}(y) g_{\mu \nu}(x)
$$

and an additional condition [the $y y$-component of Eq. (27)],

$$
C_{3}(y)+C_{4}(y) R(x)+C_{5}(y) L_{G B}(x)=0,
$$

where

$$
\begin{aligned}
& C_{1}(y)=1+4 \alpha\left(A^{\prime \prime}-A^{2}\right), \\
& C_{2}(y)=\left[\Lambda_{5}-3\left(A^{\prime \prime}-2 A^{\prime 2}\right)+12 \alpha A^{2}\left(A^{\prime \prime}-A^{\prime 2}\right)\right] e^{-2 A}, \\
& C_{3}(y)=\Lambda_{5}+6 A^{\prime 2}\left(1-2 \alpha A^{\prime 2}\right) \\
& C_{4}(y)=-\left(\frac{1}{2}-2 \alpha A^{\prime 2}\right) e^{2 A} \\
& C_{5}(y)=-\frac{\alpha}{2} e^{4 A}
\end{aligned}
$$

By dividing equation (30) by $C_{5}(y)$ and differentiating with respect to $y$, we obtain

$$
\left(\frac{C_{3}(y)}{C_{5}(y)}\right)^{\prime}-\left(\frac{C_{4}(y)}{C_{5}(y)}\right)^{\prime}(4) R(x)=0 .
$$

Now, differentiating it with respect to any brane coordinate $x$, we arrive at

$$
\left[\frac{C_{4}(y)}{C_{5}(y)}\right]^{\prime} R(x)_{, \mu}=0
$$

so that either $R(x)_{, \mu}=0$ or $\left(C_{4} / C_{5}\right)^{\prime}=0$. We call the first case physical because it has a well defined limit when $\alpha$ tends to zero, the Einsteinian limit. Einstein-Gauss-Bonnet theory is ambiguous from a dynamical point of view. The Lagrangian is quartic in first derivatives of the metric and thus the same initial data can give rise to different evolutions [23]. To resolve this ambiguity, one possibility is to choose as the physical branch that approaching proper general relativity in the limit $\alpha=0$ [24]. The second case, without an Einsteinian limit, we call "purely" Gauss-Bonnet. 
B. Physical case: $R_{, \mu}=0$

We write the constant Ricci scalar as

$$
R= \pm \frac{12}{L^{2}}
$$

By Eq. (30), $L_{G B}$ is also a constant, $L_{G B}=S_{1}$, and then Eq. (30) gives

$$
6 A^{\prime 2}\left(1-2 \alpha A^{\prime 2}\right)-\left(\frac{1}{2}-2 \alpha A^{\prime 2}\right) e^{2 A}\left( \pm \frac{12}{L^{2}}\right)-\frac{\alpha}{2} e^{4 A} S_{1}+\Lambda_{5}=0
$$

or re-arranging,

$$
2 \alpha A^{\prime 4}-\left(1 \pm \frac{4 \alpha}{L^{2}}\right) A^{\prime 2}+\left(-\frac{\Lambda_{5}}{6} \pm \frac{1}{L^{2}} e^{2 A}+\frac{\alpha}{12} e^{4 A} S_{1}\right)=0 .
$$

Multiplying Eq. (39) by $e^{-4 A}$ and differentiating we see that equations (29) can be re-expressed as proper Einstein equations for the 4-dimensional geometry,

$$
G_{\mu \nu}(x)=\mp \frac{3}{L^{2}} g_{\mu \nu}(x) .
$$

In summary, for a metric of the form Eq. (5) to be a solution of Einstein-Gauss-Bonnet theory in five dimensions, the 4-dimensional metric $g_{\mu \nu}$ must be an Einstein metric. Moreover, and this is the important point, it must have a constant Gauss-Bonnet term, i.e. $L_{G B}=$ constant. These conditions imply that the Krestschmann scalar, or equivalently in this case the square of the 4-dimensional Weyl curvature, has to be constant. Their values in terms of the constants previously introduced are

$$
R^{\mu \nu \gamma \sigma} R_{\mu \nu \gamma \sigma}=S_{1}, \quad C^{\mu \nu \gamma \sigma} C_{\mu \nu \gamma \sigma}=S_{1}-\frac{24}{L^{4}} .
$$

This condition precludes the existence of stacking solutions of the black-string type [19] and most of the solutions of astrophysical interest. The trivial conformal nature of maximally symmetric and pp-wave spacetimes allows them to be stacked to form 5-dimensional bulk solutions and subsequently braneworld models.

For arbitrary values of the constant $S_{1}$ the solution of Eq. (40) cannot be expressed in closed form. However, for the particular value $S_{1}=24 / L^{4}$, which is the relevant value for stacking maximally symmetric metrics on the brane, closed-form solutions can be given. For $0>\Lambda_{5}=-6 / l^{2}$,

$$
A^{\prime 2}=\frac{1}{4 \alpha}\left(1 \pm \frac{4 \alpha}{L^{2}} e^{2 A}+\sigma \sqrt{1-\frac{8 \alpha}{l^{2}}}\right)
$$

where $\sigma= \pm 1$. This expression has a well defined limit for $\alpha \rightarrow 0$ only when $\sigma=-1$. For this case and $0 \leq \alpha \leq l^{2} / 8$, we can define $\tilde{l}$ and $\tilde{L}$ as

$$
\begin{aligned}
\frac{1}{\tilde{l}^{2}} & =\frac{1}{4 \alpha}\left(1-\sqrt{1-\frac{8 \alpha}{l^{2}}}\right), \\
\frac{1}{\tilde{L}^{2}} & =\frac{4 \alpha}{L^{2}} .
\end{aligned}
$$

Then, substituting $A=-\ln B$, Eq. (43) is seen to be equivalent to Eq. (15), i.e.,

$$
B^{\prime 2}=\frac{B^{2}}{\tilde{l}^{2}} \pm \frac{1}{\tilde{L}^{2}}
$$

Therefore the warp factors for the Einstein-Gauss-Bonnet theory are formally equal to those discussed in Sec. [II but with re-defined values of the curvature of the stacking hypersurfaces and the bulk curvature.

Solutions with $\alpha<0$ are completely equivalent ${ }^{2}$. Their only peculiarity is that positively-curved and negativelycurved stacking hypersurfaces interchange their associated warp factors.

\footnotetext{
${ }^{2}$ In the Gauss-Bonnet modification to General Relativity arising from string theory, $\alpha$ must be positive. From this viewpoint, these solutions are not physical.
} 
The solutions for the case $\sigma=1$ are of purely Einstein-Gauss-Bonnet type in the sense that they do not have a well defined Einsteinian limit. Particularly interesting is the fact that in this case the Gauss-Bonnet term can produce a negatively curved bulk even in the absence of a bulk cosmological constant [7]. Here we take the view that these solutions are unphysical. However, a definitive analysis of their physical nature should consider not their proximity to 5-dimensional Einstein solutions, but their proximity to 4-dimensional Einstein solutions in the reduced 4-dimensional theory that emerges once the compactification (exotic or not) has taken place. This is beyond the scope of the present paper.

\section{Purely Gauss-Bonnet case}

This case is characterized by $\left(C_{4} / C_{5}\right)^{\prime}=0$, so that condition (37) is satisfied. Therefore,

$$
\frac{C_{4}(y)}{C_{5}(y)}=\left(\frac{1}{\alpha}-4 A^{\prime 2}\right) e^{-2 A}=S_{2},
$$

where $S_{2}$ is a constant. From Eq. (30),

$$
\frac{C_{3}(y)}{C_{5}(y)}+S_{2} R(x)+L_{G B}(x)=0
$$

and therefore

$$
\begin{aligned}
& \frac{C_{3}(y)}{C_{5}(y)}=-\frac{2}{\alpha} e^{-4 A}\left[\Lambda_{5}+6 A^{\prime 2}\left(1-2 \alpha A^{\prime 2}\right)\right]=S_{3}, \\
& S_{2} R(x)+L_{G B}(x)=S_{3},
\end{aligned}
$$

with $S_{3}$ another constant. By Eqs. (47) and (49), we deduce that there are two cases:

1) $S_{2}^{2}=-\frac{2}{3} S_{3} \neq 0, \Lambda_{5}=-3 / 4 \alpha$.

Again, for $\alpha>0$ we can define constants $\bar{l}$ and $\bar{L}$ via

$$
\begin{gathered}
\frac{1}{\overline{\bar{l}^{2}}}=\frac{1}{4 \alpha}, \\
\frac{1}{\bar{L}^{2}}=\frac{\left|S_{2}\right|}{4} .
\end{gathered}
$$

Setting $A=-\ln B$ we arrive at an equation equivalent to Eq. (15),

$$
B^{\prime 2}=\frac{B^{2}}{\bar{l}^{2}}+\operatorname{sign}\left(S_{1}\right) \frac{1}{\bar{L}^{2}},
$$

and therefore, to the same formal solutions for the warp factors. For these warp factors, we can check that $C_{1}(y)=$ $C_{2}(y)=0$ and so we reach the intriguing result that there is a single equation for the 4-dimensional metric

$$
S_{2} R(x)+L_{G B}(x)=-\frac{3}{2} S_{2}^{2} .
$$

2) $A(y)=$ constant.

Without lost of generality we can set $A(y)=0$, and then $S_{2}=1 / \alpha$ and $S_{3}=2 \Lambda_{5} / \alpha$. In this case the reduced set of equations to solve is, by Eqs. (29) and (49),

$$
G_{\mu \nu}=-\Lambda_{5} g_{\mu \nu}, \quad L_{G B}=-\frac{2 \Lambda_{5}}{\alpha} .
$$

If moreover $\Lambda_{5}=0$, these equations do not depend on $\alpha$ and therefore, this case does in fact have a well defined Einsteinian limit. 


\section{DISCUSSION AND CONCLUSIONS}

We have seen that in the presence of a Gauss-Bonnet term the generation of 5-dimensional bulk solutions via simple stacking of 4-dimensional metrics does not hold as may have been naively expected. Although the Gauss-Bonnet term is a topological invariant in four dimensions, in the process of reducing the system from five dimensions to four dimensions the dynamical degrees of freedom of the Gauss-Bonnet term in five dimensions leave their traces in the reduced theory (cf. 25]).

Let us consider a different point of view. In the simplest case, with zero bulk cosmological constant and no warp factor $(A=0)$, we substitute the ansatz $d s^{2}=d y^{2}+g_{\mu \nu}(x) d x^{\mu} d x^{\nu}$ into the 5-dimensional action,

$$
S=\frac{1}{2 \kappa_{5}^{2}} \int d x^{5} \sqrt{-{ }^{(5)} g}\left[{ }^{(5)} R+\alpha^{(5)} L_{G B}\right]
$$

and integrate over $y$ (one can consider a cylindrical condition on $y$ to obtain a finite result.) In this way one finds a reduced action in four dimensions of the Einstein-Hilbert form,

$$
S=\frac{1}{2 \tilde{\kappa}_{5}^{2}} \int d x^{4} \sqrt{-g} R
$$

(The trivial additive constant left by the Gauss-Bonnet term has not been written. The Gauss-Bonnet term has become topological in the reduction process.) This is the standard Kaluza-Klein reduction in the absence of the electromagnetic part of the metric (in Kaluza-Klein terminology); therefore we recover vacuum Einstein gravity in four dimensions 26]. In standard Kaluza-Klein reduction, the 5-dimensional Gauss-Bonnet term modifies the way in which the electromagnetic field interacts with gravity [27], but in the absence of this field, there is no other higher-dimensional manifestation of the Gauss-Bonnet term. However, what we have seen is that if one uses first the action Eq. (56) to obtain the 5-dimensional equations of motion, and then one specializes to the above metric ansatz, one does recover the 4-dimensional Einstein equations $R_{\mu \nu}=0$, but in addition, one finds the condition $L_{G B}=0$. This condition puts a strong restriction on the allowed geometries. Actually, this condition is equivalent to $C_{\mu \nu \rho \sigma} C^{\mu \nu \rho \sigma}=0$, so it only permits the existence of conformally trivial 4-geometries of this type. Therefore, in the presence of a Gauss-Bonnet term, the process of dimensional reduction of the action and subsequent variation is not equivalent to the process of first varying the 5-dimensional action and then reducing dimensionally the resulting equations.

What we have seen in this paper is that this phenomenon is general and shows up in braneworld (exotic) compactifications as well. Our simple ansatz does not allow any curvature singularity on the brane, in particular ruling out black string type braneworlds. In this sense our result is in tune with the well known idea that Gauss-Bonnet corrections to Einstein relativity might smooth out the singularities. Trying to find non-trivial brane geometries would involve the consideration of an electromagnetic and/ or a dilaton part for the metric with their corresponding effects.

\section{Acknowledgments}

We thank Cristiano Germani and Bruce Bassett for useful discussions and comments. CB is supported by the EC under contract HPMF-CT-2001-01203. RM is supported by PPARC. CFS and FV are supported by the EPSRC.

[1] D. Lovelock, "The Einstein tensor and its generalizations," J. Math. Phys. 12, 498, (1971).

[2] L. Randall and R. Sundrum, "A large mass hierarchy from a small extra dimension," Phys. Rev. Lett. 83, 3370, (1999). arXiv:hep-ph/9905221.

[3] L. Randall and R. Sundrum, "An alternative to compactification," Phys. Rev. Lett. 83, 4690 (1999). arXiv:hep-th/9906064.

[4] B. Zwiebach, "Curvature squared terms and string theories," Phys. Lett. B 156, 315 (1985).

[5] D. G. Boulware and S. Deser, "String generated gravity models," Phys. Rev. Lett. 55, 2656 (1985).

[6] J. E. Kim, B. Kyae and H. M. Lee, "Effective Gauss-Bonnet interaction in Randall-Sundrum compactification," Phys. Rev. D 62, 045013 (2000). arXiv:hep-ph/9912344.

[7] K. A. Meissner and M. Olechowski, "Domain walls without cosmological constant in higher order gravity," Phys. Rev. Lett. 86, 3708 (2001). arXiv:hep-th/0009122.

[8] J. E. Kim, B. Kyae and H. M. Lee, "Various modified solutions of the Randall-Sundrum model with the Gauss-Bonnet interaction," Nucl. Phys. B 582, 296 (2000) [Erratum-ibid. B 591, 587 (2000)] arXiv:hep-th/0004005. 
[9] K. A. Meissner and M. Olechowski, "Brane localization of gravity in higher derivative theory," Phys. Rev. D 65, 064017 (2002). arXiv:hep-th/0106203.

[10] C. Csaki, J. Erlich, C. Grojean and T. J. Hollowood, "General properties of the self-tuning domain wall approach to the cosmologic al constant problem," Nucl. Phys. B 584, 359 (2000). arXiv:hep-th/0004133.

[11] P. Binetruy, C. Charmousis, S. C. Davis and J. F. Dufaux, "Avoidance of naked singularities in dilatonic brane world scenarios with a Gauss-Bonnet term," Phys. Lett. B 544, 183 (2002). arXiv:hep-th/0206089.

[12] N. Deruelle and T. Dolezel, "Brane versus shell cosmologies in Einstein and Einstein-Gauss-Bonnet theories," Phys. Rev. D 62, 103502 (2000). arXiv:gr-qc/0004021.

[13] B. Abdesselam and N. Mohammedi, "Brane world cosmology with Gauss-Bonnet interaction," Phys. Rev. D 65, 084018 (2002). arXiv:hep-th/0110143.

[14] C. Germani and C. F. Sopuerta, "String inspired braneworld cosmology," Phys. Rev. Lett. 88, 231101 (2002). arXiv:hep-th/0202060.

[15] C. Charmousis and J. F. Dufaux, "General Gauss-Bonnet brane cosmology," Class. Quant. Grav. 19, 4671 (2002). arXiv:hep-th/0202107.

[16] S. Nojiri, S. D. Odintsov and S. Ogushi, "Friedmann-Robertson-Walker brane cosmological equations from the fivedimensional bulk (A)dS black hole," Int. J. Mod. Phys. A 17, 4809 (2002). arXiv:hep-th/0205187.

[17] J. E. Lidsey, S. Nojiri and S. D. Odintsov, "Braneworld cosmology in (anti)-de Sitter Einstein-Gauss-Bonnet-Maxwell gravity," JHEP 0206, 026 (2002). arXiv:hep-th/0202198.

[18] D. Brecher and M. J. Perry, "Ricci-flat branes," Nucl. Phys. B 566, 151 (2000). arXiv:hep-th/9908018.

[19] A. Chamblin, S. W. Hawking and H. S. Reall, "Brane-world black holes," Phys. Rev. D 61, 065007 (2000) arXiv:hep-th/9909205.

[20] A. Chamblin and G. W. Gibbons, "Nonlinear supergravity on a brane without compactification," Phys. Rev. Lett. 84, 1090 (2000). arXiv:hep-th/9909130.

[21] E. Anderson and J. E. Lidsey, "Embeddings in non-vacuum spacetimes," Class. Quant. Grav. 18, 4831 (2001). arXiv:gr-qc/0106090.

[22] O. DeWolfe, D. Z. Freedman, S. S. Gubser and A. Karch, "Modeling the fifth dimension with scalars and gravity," Phys. Rev. D 62, 046008 (2000).

[23] M. Henneaux, C. Teitelboim and J. Zanelli, "Quantum mechanics for multivalued Hamiltonians" Phys. Rev. A 36, 4417 (1987).

[24] G. A. Mena Marugán, "Classical And Quantum Lovelock Cosmology," Phys. Rev. D 42, 2607 (1990).

[25] M. Bañados, "General relativity from five dimensional Chern-Simons theory," Phys. Rev. D 55, 2051 (1997) arXiv:gr-qc/9603029.

[26] F. Müller-Hoissen, "From Chern-Simons to Gauss-Bonnet," Nucl. Phys. B 346, 235 (1990).

[27] H. H. Soleng and O. Gron, "Modification of the Coulomb potential from Kaluza-Klein model with a Gauss-Bonnet term in the action," Annals Phys. 240, 432 (1995). arXiv:gr-qc/9409060. 